\title{
Probiotics and their Metabolites Reduce Oxidative Stress in Middle-Aged Mice
}

\author{
Wen-Yang Lin ${ }^{1} \cdot$ Jia-Hung Lin ${ }^{1} \cdot$ Yi-Wei Kuo ${ }^{1}$ Pei-Fang Rose Chiang ${ }^{2} \cdot$ Hsieh-Hsun $\mathrm{Ho}^{1}{ }^{\circledR}$
}

Received: 9 September 2021 / Accepted: 23 January 2022 / Published online: 14 February 2022

(c) The Author(s) 2022

\begin{abstract}
Aging is an irreversible physiological degradation of living organisms. Accumulated oxidative stress and dysbiosis accelerate aging. Probiotics such as Lactobacillus and Bifidobacterium and their fermented metabolites (postbiotics) have been discovered to exhibit antioxidative activities that regulate oxidative stress and protect cells from oxidative damage. We screened selected Lactobacillus and Bifidobacterium strains and their postbiotics for potential antioxidative activity by using DPPH (2,2-Diphenyl-1-picrylhydrazyl) assay. Strains with their metabolites were selected for mixed formula in experiments involving aging mice. The aged groups presented higher oxidative stress in the brain, liver, heart, and kidney than did young mice. However, treatment with probiotic strains and their postbiotics elevated antioxidative levels, especially in the high-dose probiotics plus postbiotics group. Next-generation sequencing data revealed positive microbiota alterations of Lactobacillus and Bifidobacterium and Akkermansia in the gut. Lactobacillus johnsonii and Akkermansia muciniphila exhibited effective enlargement of relative abundance. Besides, high-dose probiotics and high-dose probiotics plus postbiotics showed significant elevation in serum SCFAs, especially in butyrate. In conclusion, the formula containing Bifidobacterium animalis subsp. infantis BLI-02, Bifidobacterium breve Bv889, Bifidobacterium bifidum VDD088, B. animalis subsp. lactis CP-9, and Lactobacillus plantarum PL-02 and their metabolites may benefit aged people's health.
\end{abstract}

\section{Introduction}

Aging is a general, progressive, cumulative, and harmful physiological decline. Because of differences between individuals, aging cannot be described with a single or simple model [1]. However, as research progresses, researchers have discovered that human aging is closely related to reactive

Hsieh-Hsun Ho

sam.ho@bioflag.com.tw

Wen-Yang Lin

wen-yang.lin@bioflag.com.tw

Jia-Hung Lin

jiahung.lin@bioflag.com.tw

Yi-Wei Kuo

vic.kuo@bioflag.com.tw

Pei-Fang Rose Chiang

rosepfpaper@gmail.com

1 Department of Research and Design, Bioflag Biotech Co., Ltd., 4F.C2, No.17, Guoji Rd, Xinshi Dist, Tainan City 744, Taiwan

2 Department of Psychology, Jacobs University Bremen, Campus Ring 1, Vegesack, 28759 Bremen, Germany oxygen species in the body [2]. Oxidative free radicals can cause damage to DNA, proteins, lipids, and other molecules of cells, causing the gradual loss of physiological functions and diseases including cardiovascular disease and cancer [3]. Numerous studies have demonstrated that oxidative free radicals are a main factor in aging in internal organs [4]. Therefore, research on new antioxidant substances that delay aging is the principal focus of antiaging research.

Over $10^{14}$ microorganisms inhabit the human gastrointestinal (GI) tract. The number of GI bacterial cells is 10 times that of human cells, with microbial genomic content numbering over 3 million, whereas the human genome contains approximately 23,000 genes [5]. Scientists have discovered microbiota changes in older people [6], whose number of beneficial gut bacteria, including Lactobacilli and Bifidobacteria, are greatly reduced and certain facultative anaerobes and gram-negative bacteria (mainly Enterobacteria) multiply. The shift of gut microbiota may lead to small bowel bacterial overgrowth followed by symptoms such as diarrhea, nutrient malabsorption, and weight loss in aged people [7].

Accumulated findings have demonstrated that probiotic strains of Lactobacilli and Bifidobacteria possess antioxidant properties [8]. The metabolites secreted by probiotic 
strains (postbiotics) may play key roles in promoting antioxidative activity. An animal study confirmed that probiotic metabolites improve serum antioxidant activity and upregulate hepatic antioxidant enzymes [9]. Moreover, a doubleblind clinical trial revealed that probiotic supplementation in patients with Alzheimer disease improved cognitive function [10]. Moreover, the food safety of lactic acid bacteria has been validated in clinical trials [11]. However, unpredicted side effects have been reported in some effective antioxidants, such as resveratrol. For example, in clinical trials, taking high doses of resveratrol (2.5-5.0 g/day) led to symptoms such as nausea, flatulence, abdominal discomfort, and diarrhea [12]. Anticoagulant effects were also revealed, and it may interfere with the metabolism of drugs in the liver [13].

Probiotic strains together with their fermented products can be considered candidate nutritional supplements for downregulating the oxidative stress that induces the aging process. DPPH (2,2-diphenyl-1-picrylhydrazyl) is an oxidative free radical, which has been widely used to select antioxidative substances or organisms before animal study [14, 15]. In this study, we screened effective antiaging probiotic strains through in vitro antioxidative assay (DPPH assay). We then used an animal model to validate the antioxidative properties of the probiotic strains with their metabolites. Finally, the microbiota shifts caused by taking the probiotic products were detected and confirmed using the next generation sequencing technique (NGS).

\section{Materials and Methods}

\section{Probiotic Strains and Culturing of Strains}

All the probiotic strains were sourced from human intestinal tract and obtained from Bioflag Biotech Co. (Tainan, Taiwan). The strains were preserved in the China Center for Type Culture Collection (CCTCC) and the China General Microbiological Culture Collection Center (CGMCC). The deposit numbers for Bifidobacterium animalis subsp. infantis BLI-02, Bifidobacterium breve Bv889, Bifidobacterium bifidum VDD088, B. animalis subsp. lactis CP-9, and Lactobacillus plantarum PL-02 are CGMCC-15212, CGMCC16145, CGMCC-15211, CCTCC-M2014588, and CGMCC20485, respectively. Lactobacillus lactis L-87, Lactobacillus rhamnosus L-13, Enterococcus faecium L-38, Lactobacillus gasseri L-2, Lactobacillus paracasei L-134, L. paracasei L-30 and Streptococcus thermophilus L-243 were obtained from Bioflag Biotech Co. (Tainan, Taiwan).

The probiotic lactic acid bacteria strains were stored at $-80^{\circ} \mathrm{C}$ with $20 \%$ glycerol. MRS broth (Difco, BD ${ }^{\mathrm{TM}}$, New Jersey, United States) containing $0.05 \%$ cysteine was used to activate the strains at $37^{\circ} \mathrm{C}(24 \mathrm{~h})$ twice. The postbiotic products were gained from fermentation of the strains. A liquid medium of 5\%-30\% milk and $1 \%-10 \%$ soybean meal was used for fermenting the probiotics. The fermentation broth was finally purified through centrifugation, filtration, and heat sterilization and dried into powder. The powder of the fermentation broth was stored at room temperature.

\section{Screening Potential Probiotic Strains Through Antioxidative Assay}

DPPH (2,2-Diphenyl-1-picrylhydrazyl) is a stable free radical molecule whose highest absorption value is at a wavelength of $517 \mathrm{~nm}$ in a methanol solution. When DPPH free radicals interact with antioxidants, the antioxidants provide hydrogen protons to scavenge free radicals, and DPPH free radicals lose their blue-violet characteristics and their light absorption decreases. The decrease in $\mathrm{OD}_{517}$ value was used to determine the free radical scavenging ability of the tested lactic acid bacteria strains.

The method for detecting the free radical scavenging ability of the lactic acid bacteria strains or their metabolites was as follows. Common lactic acid bacteria strains (approximately $2 \times 10^{9}$ colony-forming units [CFU], optical density [OD] approximately 2 ) or a strain's own fermentation broth were mixed $1: 1$ with $0.2 \mathrm{mM}$ DPPH in methanol. After being mixed, the solution was maintained at room temperature in the dark for $30 \mathrm{~min}$ for reaction. After centrifuging $(12,000 \mathrm{rpm}, 2 \mathrm{~min})$ at $4{ }^{\circ} \mathrm{C}$ and transferring $200 \mu \mathrm{L}$ of supernatant to a 96 -well plate, the $\mathrm{OD}_{517}$ value was measured. The Streptococcus thermophiles SY-66 strain without antioxidant activity was used as a negative control (approximately $2 \times 10^{9} \mathrm{CFU}$, OD approximately 2) and diluted water as a blank control. The calculation formula used for free radical scavenging ability is as follows:

Free radical scavenging capacity

$$
=\mathrm{OD}_{\text {blank }}-\mathrm{OD}_{\text {sample }} / \mathrm{OD}_{\text {blank }} \times 100 \%
$$

where $\mathrm{OD}_{\text {sample }}$ is the absorbance value of the tested sample and $\mathrm{OD}_{\text {blank }}$ is the absorbance value of the blank group. Vita$\min \mathrm{C}(10 \mu \mathrm{g} / \mathrm{mL})$ was used as a positive control.

\section{Mice and Ethics Statement}

Animal experiments and protocols were in compliance with the National Institute of Health's Guide for the Care and Use of Laboratory Animals. The protocols were approved (the Approval Number-IACUC no.NLAC(TN)-108-D-002) by animal ethics committee of National Laboratory Animal Center (Taipei, Taiwan). C57BL/6 mice (age: 2 months) were used for the study. The animals were housed in groups of six in sterilized cages fitted with filter cage tops and fed 
with sterilized food and water. The housing environment was strictly monitored and maintained under $22 \pm 2{ }^{\circ} \mathrm{C}$ and $62 \% \pm 5 \%$ humidity. Each group was entrusted to the National Laboratory Animal Center (Taipei, Taiwan) during the experiment, and tube feeding was administered daily according to the experimental design.

\section{Animal Experimental Design}

The experimental mice naturally developed and were divided into nine groups (each group with four mice, two male plus two female) as follows: 2-month-old (2 M), 10-month-old (10 M), 13-month-old (13 M), and 16-month-old (16 M) groups; among the 10-month-old groups were a low-dose probiotics group $\left(1.03 \times 10^{9} \mathrm{CFU} / \mathrm{kg}\right.$, per mouse, daily dose), a low-dose probiotics and postbiotics group $\left(1.03 \times 10^{9} \mathrm{CFU} /\right.$ $\mathrm{kg}$ plus $20.5 \mathrm{mg} / \mathrm{kg}$ of postbiotics, per mouse, daily dose), a high-dose probiotics group $\left(4.1 \times 10^{9} \mathrm{CFU} / \mathrm{kg}\right.$, each mouse, daily dose), a high-dose probiotics and postbiotics group $\left(4.1 \times 10^{9} \mathrm{CFU} / \mathrm{kg}\right.$ probiotics plus $20.5 \mathrm{mg} / \mathrm{kg}$ of postbiotics, per mouse, daily dose), and a positive control group fed general feed plus resveratrol ( $25 \mathrm{mg} / \mathrm{kg}$ mice/day). The $2 \mathrm{M}$, $10 \mathrm{M}, 13 \mathrm{M}$, and $16 \mathrm{M}$ groups were fed with general feed without extra treatment. The mice were sacrificed for the detection of oxidative and antioxidative elements in tissues.

The probiotic-treated groups and the positive control group were fed with treatments from the age of 10 months to 16 months. The dose of lactic acid bacteria ingested by the animals was estimated according to the initial experimental method published by the US Food and Drug Administration in 2005. The five probiotic strains were mixed at a ratio of 1:1:1:1:1 for daily feeding, and the postbiotics were mixed with the same five strains in the same ratio. After consecutive feedings for 6 months, the tissues of this "aging" group were also evaluated (Supplemental Fig. 1).

\section{Evaluating Oxidative and Antioxidative Level in Mice}

All mice were sacrificed using $\mathrm{CO}_{2}$; next, the brain, heart, liver, and other tissues were removed with surgical instruments and placed in microcentrifuge tubes; $200 \mu \mathrm{L} 0.4 \mathrm{M}$ perchloric acid was added, and an ultrasonic homogenizer was used for homogenization (all procedures were performed on ice). Subsequently, the antioxidative levels in the mouse tissues were measured through superoxide dismutase (SOD) assay (Cayman Chemical Item No. 706002), glutathione peroxidase (GPx) assay (Cayman Chemical Item No. 703102), and catalase (CAT) assay (Cayman Chemical Item No. 707002). The oxidative levels were measured (and the protocol followed) through TBARS assay (Cayman Chemical Item No. 10009055), protein carbonyl colorimetric assay (Cayman Chemical Item No. 10005020), and New 8OHdG
Check ELISA (Item No. KOG-200SE, JalCA). Mitochondria DNA was extracted using a mitochondrial DNA isolation kit (Item No. K280-50, BioVision) for measuring 8-oxo2 '-deoxyguanosine (8OHdG). All assays were conducted in accordance with the experimental standard procedures provided with the product. All oxidative and antioxidative indicators are consistent with the standard experimental test for antiaging health food identified by the Ministry of Health of the Republic of China (Taiwan).

\section{Evaluating Gut Microbiota Change Through the NGS Method}

Mouse feces were collected after 6 months of consecutive treatment and immediately placed in $\mathrm{a}-80^{\circ} \mathrm{C}$ refrigerator. Subsequently, Quick-DNATM fungal/bacterial micropreparation reagent (ZYMO Research, USA) was used to extract the DNA from the stool samples. Diluent DNA in sterile water $(5 \mathrm{ng} / \mu \mathrm{L})$ was used for quality inspection through $1 \%$ agar gel electrophoresis. Next, we amplified the DNA fragments (16S rRNA, 16S V3-V4) by using polymerase chain reaction (PCR) through specific DNA-fragment targeting primers (Pusion High-Fidelity PCR Master Mix, New England Biolabs, USA). The purifying PCR generated DNA products by using AMPure XP beads (Beckman Coulter Genomics, USA). Then, an Illumina Nextera XT Index kit (Illumina, USA) was used to produce sample libraries, which were evaluated using a Qubit@2.0 Fluorometer (Thermo Scientific, USA) and sequenced on the Illumina MiSeq platform. The Greengenes database (http://greengenes.lbl. gov) was used for merging total reads, removing low-quality sequence, removing chimera sequence and clustering the OTU at $97 \%$ similarity. The CLC Microbial Genomics Module (Qiagen, Germany), basespace (illumine, USA) and Graphpad prism 8.1 (Graphpad Software, San Diego, CA, USA) were used to analyze all OTU sequences and diversity. The analysis procedure was according to previous study [16].

\section{Measurements of Serum Short-Chain Fatty Acids (SCFAs) Levels}

Add $0.3 \mathrm{~mL}$ of deionized water to $0.03 \mathrm{~g}$ of feces samples for homogenization.

Centrifuge homogenized feces samples to isolate $150 \mathrm{ul}$ of the supernatant. Add $50 \mathrm{ul}$ of $50 \%$ sulfuric acid, $10 \mathrm{ul}$ internal standard and $200 \mathrm{ul}$ ether to feces samples and shake for $15 \mathrm{~min}$. Centrifuge at $4{ }^{\circ} \mathrm{C} 9000 \mathrm{rpm}$ for $10 \mathrm{~min}$. The ether layer was added with anhydrous sodium sulfate $\left(\mathrm{Na}_{2} \mathrm{SO}_{4}\right)$ for dehydration and then detected by GS/MS (Agilent 7890 gas chromatography mass spectrometer equipped with Agilent HP-FFAP capillary column, $30 \mathrm{~m} * 250 \mathrm{um} * 0.25 \mathrm{um}$; Santa Clara, CA, United States). The GS/MS analysis conditions 
were as follows: injection volume $1 \mathrm{uL}$ (Split 5:1), gas flow rate $(1 \mathrm{~mL} / \mathrm{min})$, heating conditions (hold $80{ }^{\circ} \mathrm{C}$ for $1 \mathrm{~min}$, then raised to $150{ }^{\circ} \mathrm{C}$. Heating rate was $5^{\circ} \mathrm{C} / \mathrm{min}$. After raising temperature to $230{ }^{\circ} \mathrm{C}$, heating rate became $40{ }^{\circ} \mathrm{C} / \mathrm{min}$, hold on $12 \mathrm{~min}$ ). Injection temperature was $240{ }^{\circ} \mathrm{C}$. Transmission line temperature was $240^{\circ} \mathrm{C}$. Ion source temperature was $230{ }^{\circ} \mathrm{C}$. The temperature of the quadrupole was $150{ }^{\circ} \mathrm{C}$. The experimental protocol was followed previous study [17]. Serum SCFA level were presented as $(\%)=$ averaged SCFAs levels in treatment groups/averaged SCFAs levels in $16 \mathrm{M}$ mice control.

\section{Statistics}

The contents of each group were then analyzed (Graphpad Prism 8, Graphpad Software, San Diego, CA, USA).) using Brown-Forsythe ANOVA accompanied with Tamhane's T2 (post hoc) to compare the differences between the groups. For the NGS analysis, heatmap figures were generated using Graphpad Prism 8.1. Statistical significance was indicated if $* P<0.05, * * P<0.01, * * * P<0.001, * * * * P<0.0001$.

\section{Results}

\section{Screening Potential Probiotics Through In Vitro Antioxidative Testing}

First, we used an in vitro DPPH assay to screen probiotic strains potentially possessing antioxidative ability. The five strains with superior antioxidative ability were Bv889 (65.6\%), BLI-02 (52.8\%), PL-02 (40.2\%), CP-9 (21.6\%), and VDD088 (13.6\%). The antioxidative ability of the others was less than $10 \%$. Vitamin C $(10 \mu \mathrm{g} / \mathrm{mL} ; 91.84 \%)$ was used as the positive control for the radical scavenging test (Fig. 1a). We further tested the antioxidative ability of the postbiotics. The postbiotics exhibited excellent antioxidative ability, among which that of Bv889 was $91.2 \%$, CP-9 $86.4 \%$, Bf-668 81.9\%, BLI-02 67.3\%, and PL-02 43.6\% (Fig. 1b). Subsequently, we selected the five trains with the highest antioxidative ability and their postbiotics (Bv889, CP-9, Bf-668, BLI-02, and PL-02) to treat aging mice.

\section{Confirming the Successful Setting of Naturally Aging Mouse Model}

We used the murine model to investigate whether oxidative stress would be mitigated by probiotic treatment. Researchers have defined 2-6-month-old mice as equivalent to young human adults ( $18-30$ years), 10-16 months as human middle age ( 38-49 years), and 18-24 months as human old age ( 56-69 years) [18, 19]. We measured oxidative stress markers in mice from age 2 months to 16 months.

Because the experiment was conducted in a natural aging mouse model, observation of the change in oxidative stress indicators and antioxidant enzymes in the mice by age was necessary to confirm that the establishment of the natural aging model was successful. Therefore, prior to the start of the formal experiment, mice groups of 2 months, 10 months, 13 months, and 16 months of age were collected, and their brains, hearts, livers, and kidneys were tested for antioxidant enzymes, including SOD, CAT, and GPx, all of which gradually decline with age. We also examined a lipid, a protein, and a nucleic acid under peroxide attack by aging. The biological activity indicators included lipid oxide in propylene glycol (malondialdehyde, MDA), protein carbonyl after protein oxidation, and mitochondrial $80 \mathrm{HdG}$ content, levels of which increase with age (Fig. 2; Supplemental Figs. 2-5).

\section{Brain, Heart, Liver, Kidney Antioxidative Levels Elevated in Middle-Aged Mice Taking Probiotics}

According to the results of previous experiments, we fed different formulas of selected probiotic strains with their metabolites to 10-month-old mouse groups for 6 months (Fig. 2; Supplemental Figs. 2-5). Each group consisted of two male and female mice. The feeding groups were as described in Materials and Methods. The selected strains were mixed probiotics with strong antioxidant capacity according to prior in vitro experiments.

Oxidative stress markers MDA significantly decreased in brain, heart, liver and kidneyby taking probiotic formulas (Fig. 2a-d). Compared with the 16 months natural aging group $(4.64 \mu \mathrm{M})$, brain MDA levels decreased in all the experimental groups, including the resveratrol control $(1.78 \mu \mathrm{M}, * * P<0.01)$, low-dose probiotics $(1.82 \mu \mathrm{M}$, $* * P<0.01)$, low-dose probiotics plus postbiotics $(1.95 \mu \mathrm{M}$, $* * P<0.01)$, high-dose probiotics $(1.46 \mu \mathrm{M}, * * P<0.01)$, and high-dose probiotics plus postbiotics $(1.53 \mu \mathrm{M}$, $* * * P<0.001)$ groups (Fig. 2a). Furthermore, high-dose treatment with probiotics and high-dose probiotics plus postbiotics significantly reduced protein $\mathrm{C}+$ and mitochondrial 8OHdG levels in the brain (Supplementary Fig. 2e).

Heart MDA levels decreased in all the experimental groups by comparing to $16 \mathrm{M}$ middle-age group $(7.79 \mu \mathrm{M})$, including the resveratrol control $(4.08 \mu \mathrm{M}$, *** $P<0.001)$, low-dose probiotics $(5.59 \mu \mathrm{M})$, low-dose probiotics plus postbiotics $(4.76 \mu \mathrm{M}, * * P<0.01)$, high-dose probiotics $(5.03 \mu \mathrm{M}, * * P<0.01)$, and high-dose probiotics plus postbiotics $(5.11 \mu \mathrm{M}, * * P<0.01)$ groups (Fig. $2 \mathrm{~b})$.

Liver MDA levels decreased in all the experimental groups by comparing to $16 \mathrm{M}$ middle-age group $(4.8 \mu \mathrm{M})$, including the resveratrol control $(2.88 \mu \mathrm{M}, * * P<0.01)$, 
Fig. 1 Screening potential antioxidative probiotics through in vitro radical scavenging capacity testing (DPPH assay). a antioxidative ability ranking of viable probiotic strains. b antioxidative ability ranking of metabolites of probiotic strains (postbiotics). The experimental probiotic strains were listed below: $B$. animalis subsp. infantis $\mathrm{BLI}-02$, B. breve $\mathrm{Bv} 889$, $B$. bifidum VDD088, B. animalis subsp. lactis CP-9, Lactobacillus plantarum $\mathrm{PL}-02$, L. lactis L-87, L. rhamnosus L-13, E. faecium L-38, L. gasseri L-2, $L$. paracasei L-134, L. paracasei L-30, and S. thermophilus L-243. $10 \mu \mathrm{g} / \mathrm{ml}$ of vitamin C was used as positive control (a)
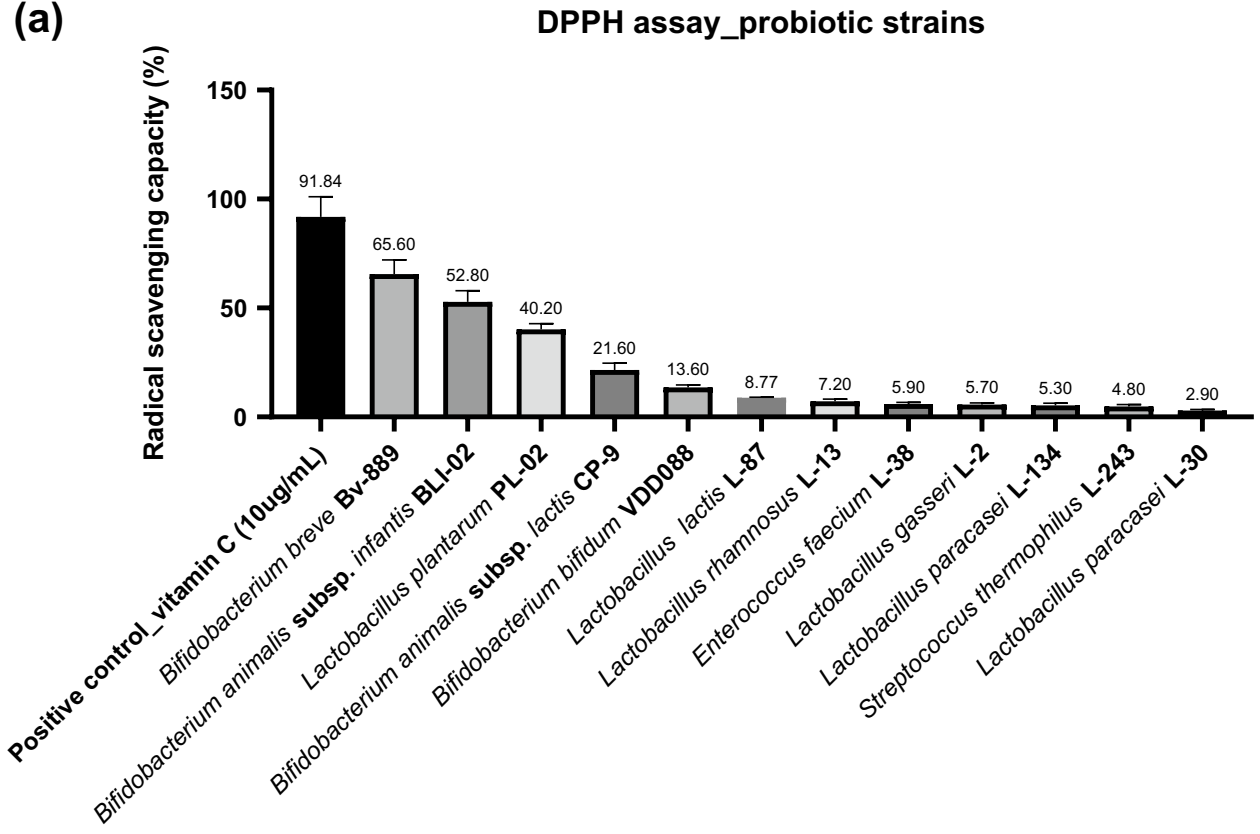

(b)

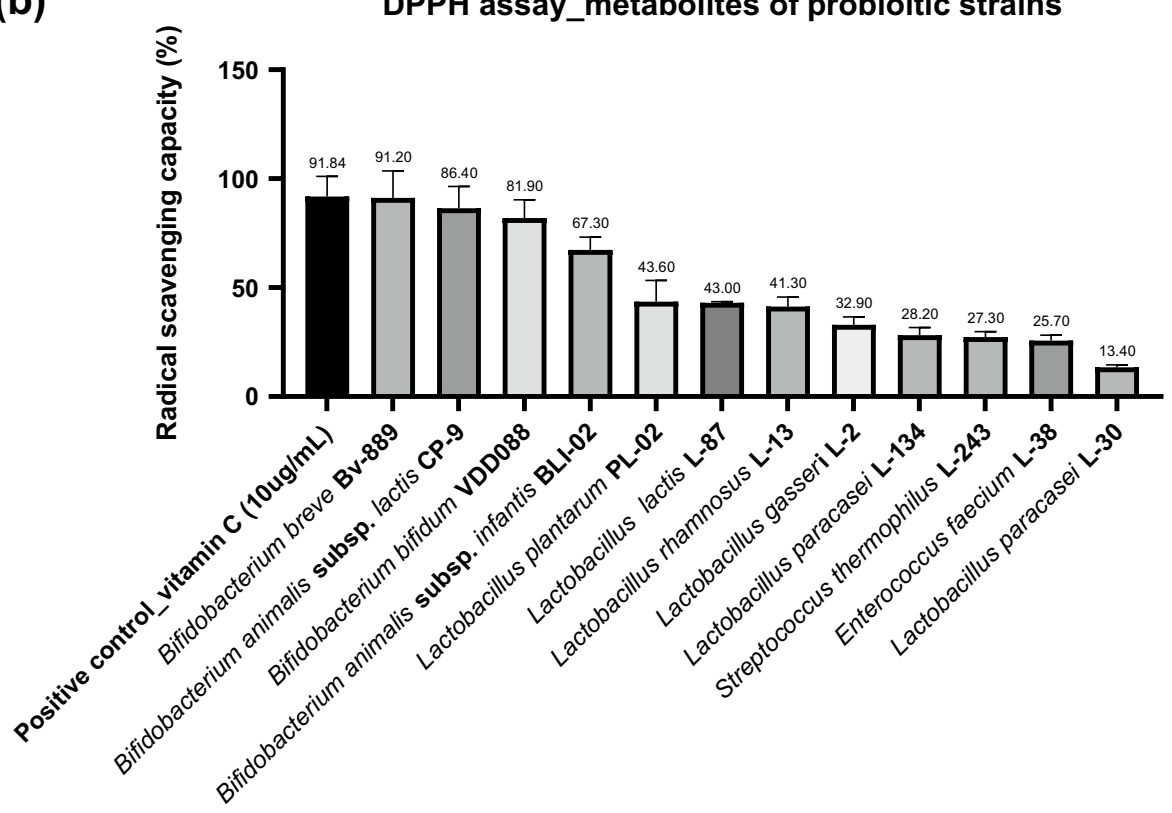

low-dose probiotics $(0.78 \mu \mathrm{M}, * * * P<0.001)$, low-dose probiotics plus postbiotics $(1.32 \mu \mathrm{M}, * * * P<0.001)$, highdose probiotics $(1.52 \mu \mathrm{M}, * * * P<0.001)$, and high-dose probiotics plus postbiotics $(1.36 \mu \mathrm{M}$, **** $P<0.0001)$ groups (Fig. 2c). Finally, kidney MDA levels decreased in all the experimental groups by comparing to $16 \mathrm{M}$ middle-age group $(3.31 \mu \mathrm{M})$, including the resveratrol control $(1.59 \mu \mathrm{M}, * * P<0.001)$, low-dose probiotics (1.6 $\mu \mathrm{M}, * * P<0.01)$, low-dose probiotics plus postbiotics $(1.72 \mu \mathrm{M}, * P<0.05)$, high-dose probiotics $(1.74 \mu \mathrm{M}$,
$* * P<0.01)$, and high-dose probiotics plus postbiotics (1.33 $\mu \mathrm{M}, * * P<0.01)$ groups (Fig. $2 \mathrm{~d}$ ).

\section{Gut Microbiota Phylum Changes Among Middle-Aged Mice Fed Probiotics}

We examined gut microbiota changes to verify whether the fed probiotics colonized in the gut and determine the influence on antioxidative levels of the colonizing probiotic strains. NGS was employed to analyze microbiota in mouse feces. We compared the microbiota of seven groups: 
(a)

Brain MDA

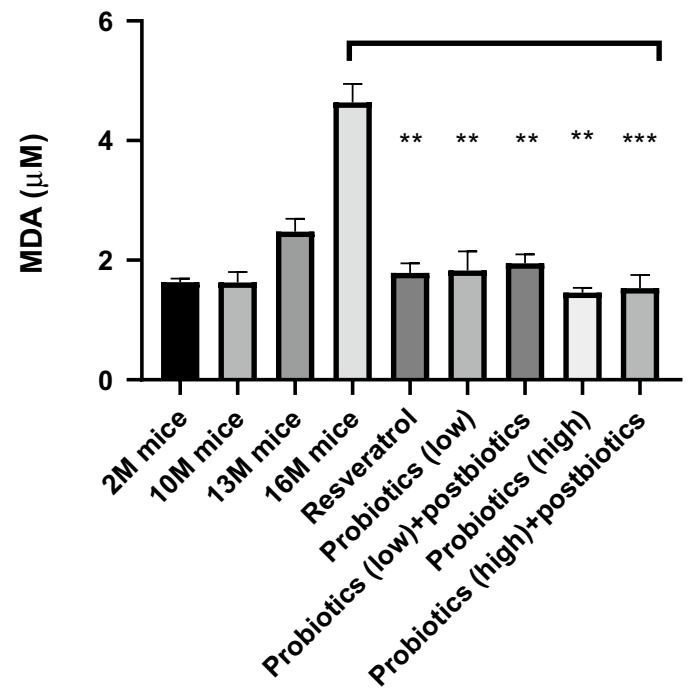

(c)

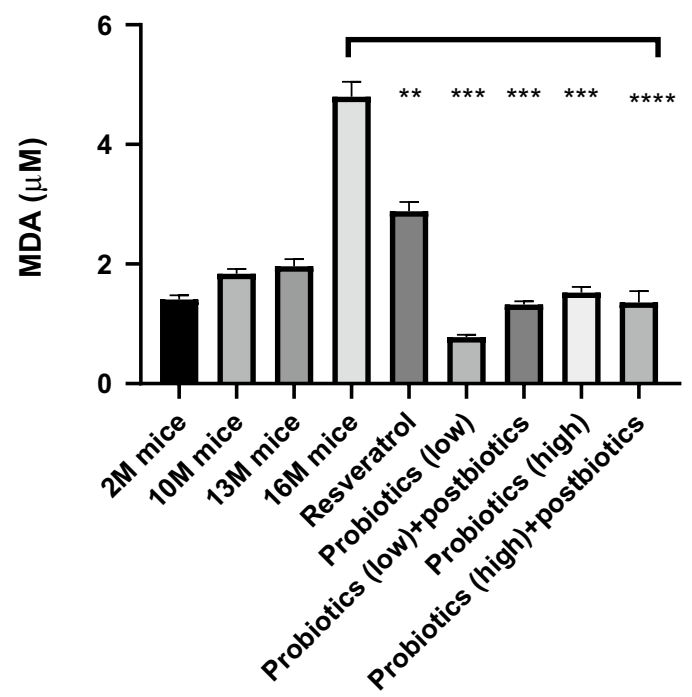

(b)

Heart MDA

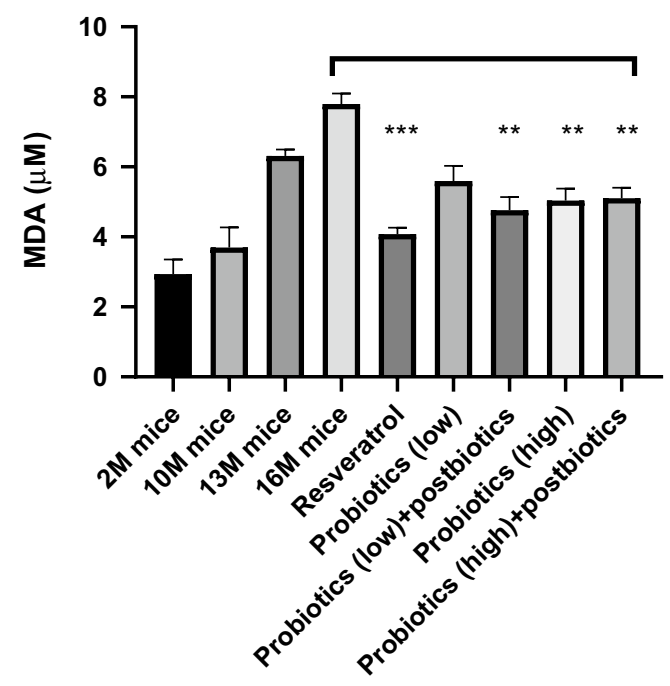

(d)

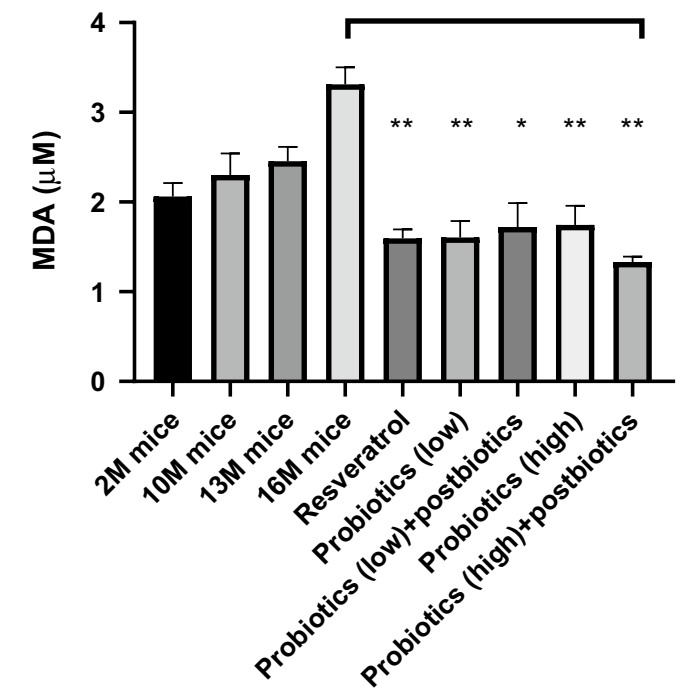

those older than 10 months were considered aged mice. Resveratrol was used as positive control for antiaging intervention. We began the probiotic treatment from the 10th month of age and treated the mice for 6 months. Treatment groups were compared with the $16 \mathrm{M}$ group (vehicle control). $* P<0.05$, $* * P<0.01, * * * P<0.001$

At the phylum level, the relative abundance of proteobacteria was significantly elevated in the high-dose probiotics plus postbiotics group $(5.35 \%, P<0.05)$ compared with the $16 \mathrm{M}$ group (1.36\%) (Fig. 3a). The relative abundance of Actinobacteria was $0.14 \%$ in $2 \mathrm{M}$ and $0.043 \%$ in $16 \mathrm{M}$. However, treatment with resveratrol, low-dose probiotics plus postbiotics, high-dose of probiotics, and highdose probiotics plus postbiotics significantly increased gut 
(a)

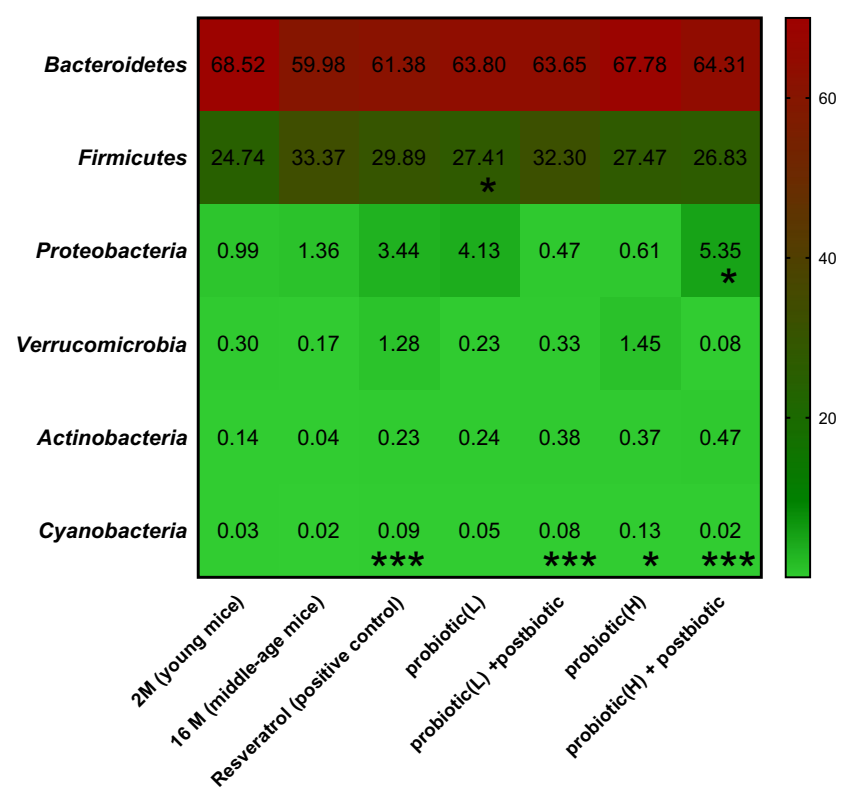

(b)

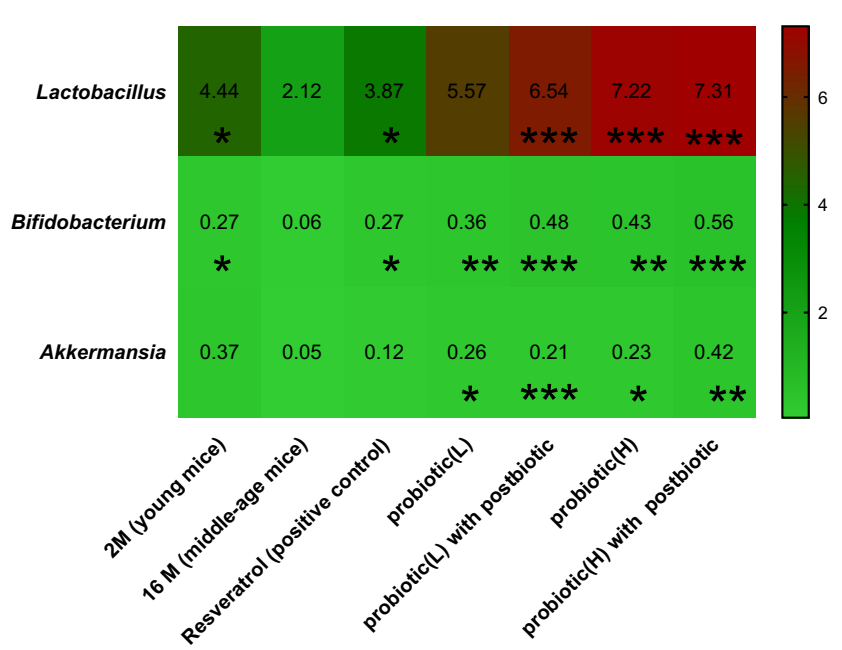

Fig. 3 NGS analysis of gut microbiota change by taking probiotic product $\mathbf{a}$ in phylum level and $\mathbf{b}$ in genus level. The group $2 \mathrm{M}$ was considered as young age mice. We start to feed probiotic treatment from 10th month and treatment last 6 months. Treatment groups were

Actinobacteria to $0.22 \%(P<0.001), 0.38 \%(P<0.001)$, $0.37 \%(P<0.05)$, and $0.46 \%(P<0.001)$, respectively (Fig. 3a).

\section{Genus Abundance of Lactobacillus, Bifidobacterium, and Akkermansia Elevated by Probiotics}

At the genus level, the relative abundance of Lactobacillus was $4.43 \%$ in $2 \mathrm{M}$, whereas it dropped significantly to $2.11 \%(P<0.05)$ in the middle-age group with natural aging (Fig. 3b). However, after 6 months of intervention, the resveratrol, low-dose probiotics, low-dose probiotics plus postbiotics, high-dose probiotics, and high-dose probiotics plus postbiotics groups had elevated gut populations of Lactobacillus of $3.86 \%(P<0.05), 5.56 \%, 6.54 \%(p<0.001), 7.22 \%$ $(P<0.001)$, and $7.31 \%(P<0.001)$, respectively (Fig. 3b). Similarly, the relative abundance of Bifidobacterium was $0.27 \%$ in the $2 \mathrm{M}$ group but was significantly decreased to $0.05 \%(P<0.05)$ in the middle-aged group. After 6 months of intervention, the resveratrol, low-dose probiotics, lowdose probiotics plus postbiotics, high-dose probiotics, and high-dose probiotics plus postbiotics groups exhibited elevated gut populations of Lactobacillus of $0.27 \%(P<0.05)$, $0.35 \%(P<0.01), 0.48 \%(P<0.001), 0.42 \%(P<0.01)$, and $0.56 \%(P<0.001)$, respectively (Fig. 3b). Furthermore, the relative abundance of Akkermansia was $0.35 \%$ in $2 \mathrm{M}$, whereas it was significantly decreased to $0.04 \%(P<0.05)$ compared to the $16 \mathrm{M}$ group (mice aged 16 months without any probiotic treatment, as vehicle control). Resveratrol was used as positive treatment control. $P<0.05^{*}, P<0.01^{* *}$ and $P<0.001^{* * *}$ stands for statistical difference

in the middle-aged group. After 6 months of intervention, the resveratrol, low-dose probiotics, low-dose probiotics plus postbiotics, high-dose probiotics, and high-dose probiotics plus postbiotics groups contained elevated gut populations of Lactobacillus of $0.11 \%, 0.26 \%(P<0.05)$, $0.2 \%(P<0.001), 0.23 \%(P<0.01)$, and $0.41 \%(P<0.01)$, respectively (Fig. 3b).

\section{Probiotic Intervention Changed the Dispersion of Lactobacillus Species in Middle-Aged Mice}

Next, The Greengenes database was used to classify the OTU of Lactobacillus and Bifidobacterium at 97\% similarity. The abundance of $L$. plantarum was significantly expanded in the probiotic-treated groups, specifically, in the low-dose probiotics $(0.01 \%, P<0.001)$, low-dose probiotics plus postbiotics $(0.02 \%, P<0.05)$, high-dose probiotics $(0.01 \%, P<0.001)$, and high-dose probiotics plus postbiotics $(0.03 \%, P<0.001)$ groups (Fig. $4 \mathrm{a})$. The abundance of Lactobacillus intestinalis, Lactobacillus japonicas, Lactobacillus pentosus, Lactobacillus reuteri, and Lactobacillus johnsonii was also significantly increased in the probiotic groups.

Among all species of Lactobacillus in the gut, L. johnsonii exhibited a dramatic change following probiotic treatment. The relative abundance of $L$. johnsonii was $3.32 \%$ in $2 \mathrm{M}$ but decreased to $1.29 \%$ in the middle-aged group. After 
(a) Lactobacillus_in species level

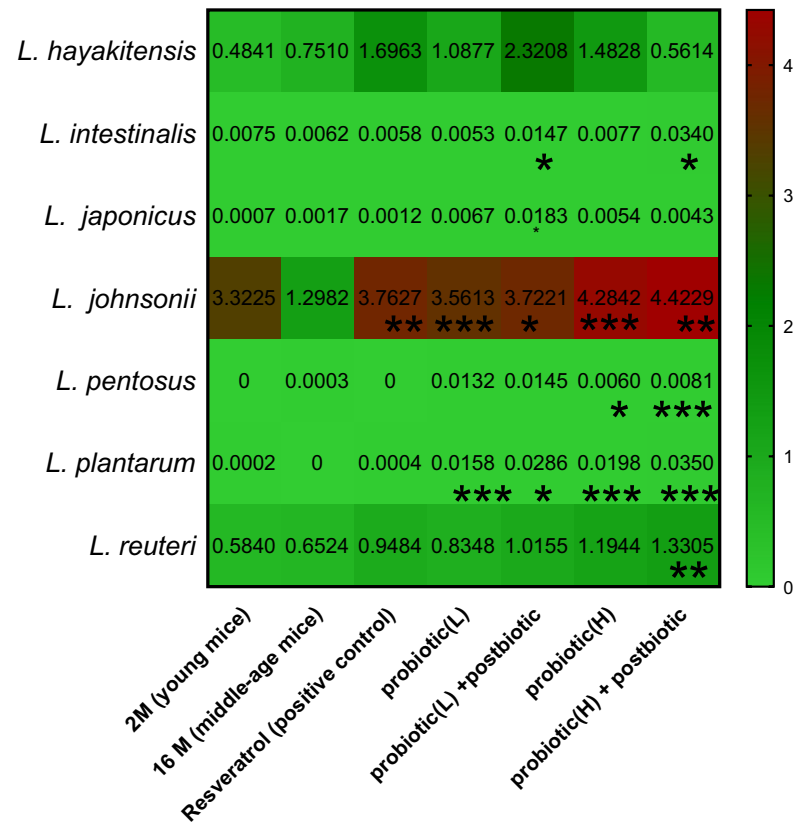

Fig. 4 NGS analysis of gut microbiota change by taking probiotic product $\mathbf{a}$ in species of Lactobacillus and $\mathbf{b}$ in species of Bifidobacterium. The group $2 \mathrm{M}$ was considered as young age mice. We start to feed probiotic treatment from 10th month and treatment last

6 months of intervention, the resveratrol, low-dose probiotics, low-dose probiotics plus postbiotics, high-dose probiotics, and high-dose probiotics plus postbiotics groups had elevated gut populations of $L$. johnsonii, of $3.76 \%(P<0.01)$, $3.56 \%(P<0.001), 3.72 \%(P<0.05), 4.28 \%(P<0.001)$, and 4.42\% $(P<0.01)$, respectively (Fig. 4a).

\section{Probiotic Intervention Changed the Dispersion of Species of Bifidobacterium in Middle-Aged Mice}

The abundance of each of $B$. animalis, B. bifidum, and $B$. breve in the gut was elevated by the mixed probiotic strains (Fig. 4b). The gut population of B. animalis significantly increased in the high-dose probiotics group $(0.0029 \%$, $P<0.01)$ and the high-dose probiotics plus postbiotics group $(0.01 \%, P<0.001)$. The abundance of gut $B$. bifi$d u m$ was significantly elevated in the low-dose probiotics plus postbiotics $(0.05 \%, P<0.001)$, high-dose probiotics $(0.04 \%, P<0.001)$, and high-dose probiotics plus postbiotics $(0.13 \%, P<0.001)$ groups. Moreover, all probiotic treatment groups had greater abundance of $B$. breve in the gut; $0.004 \%$ $(P<0.05)$ in the low-dose probiotics, $0.007 \%(P<0.05)$ in the low-dose probiotics plus postbiotics, $0.005 \%(P<0.05)$ in the high-dose probiotics, and $0.016 \%$ in the high-dose (b)

Bifidobacterium_in species level

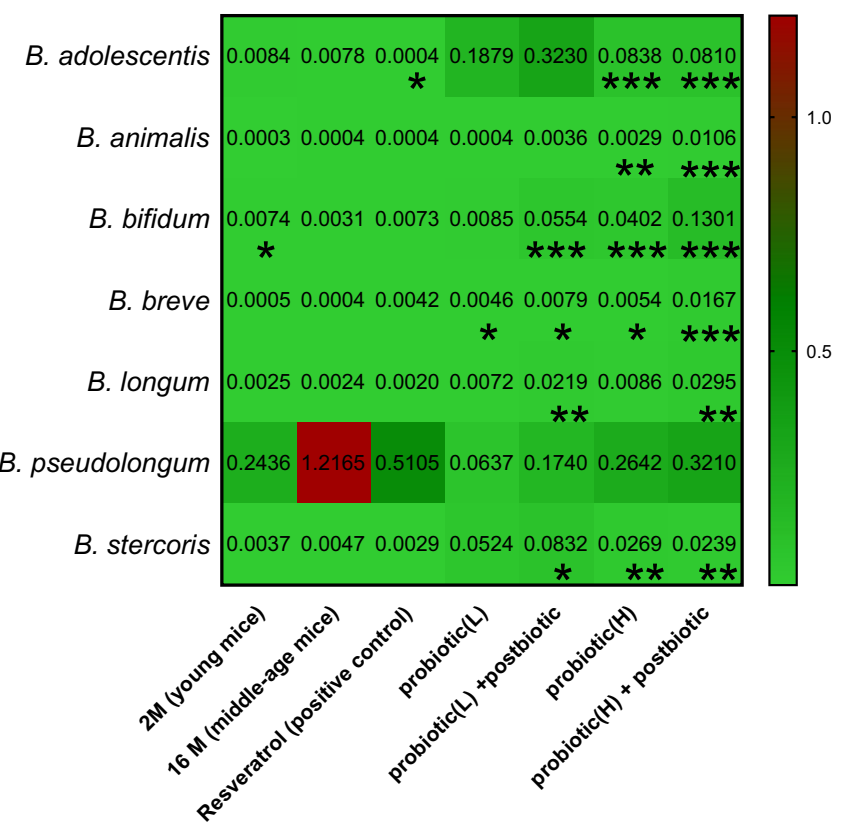

6 months. Treatment groups were compared to the $16 \mathrm{M}$ group (mice aged 16 months without any probiotic treatment, as vehicle control). Resveratrol was used as positive treatment control. $P<0.05^{*}$, $P<0.01^{* *}$ and $P<0.001^{* * *}$ stands for statistical difference

probiotics plus postbiotics $(P<0.001)$ groups (Fig. 4b). Moreover, some gut Bifidobacterium populations different from the Bifidobacterium in the supplement of mixed strains also increased after treatment, including Bifidobacterium adolescentis, Bifidobacterium longum, and Bifidobacterium stercoris.

\section{High-Dose Probiotic Intervention Changed the Serum Short-Chain Fatty Acids (SCFAs) Distribution in Middle-Aged Mice}

SCFA belongs to the nature of the metabolites produced by probiotics. Next, the serum SCFAs levels were measured among different treatment groups (Fig. 5). Comparing to non-treatment control (16 M mice), resveratrol, high-dose probiotic treatment and high-dose probiotic plus postbiotic in serum SCFAs. High-dose probiotic treatment significantly increased the serum butyrate $(478.8 \%, P<0.001)$, caproate $(135.9 \%, P<0.05)$, octanoic acid $(201.5 \%, P<0.01)$ and decanoic acid $(280.1 \%, P<0.01)$ levels. The high-dose probiotic plus postbiotic would significantly elevate serum butyrate $(518.5 \%, P<0.001)$, isovalerate $(168.9 \%, P<0.05)$ and caproate $(126.3 \%, P<0.01)$. 
Fig. 5 Serum short-chain fatty acids (SCFAs) levels in middleaged group. The group of 16 months old (16 M) was not treated with probiotics, which was considered middle-aged mice. We began the probiotic treatment from the 10th month of age and continued the treatment for 6 months. Resveratrol was used as positive control for antiaging intervention. Serum short-chain fatty acids (SCFAs) were measured after sacrificing including acetate, propionate, isobutyrate, butyrate, valerate, caproate, hexanoic acid, octanoic acid, and decanoic acid. Treatment groups were compared with the $16 \mathrm{M}$ group (vehicle control). $* P<0.05$, $* * P<0.01, * * * P<0.001$

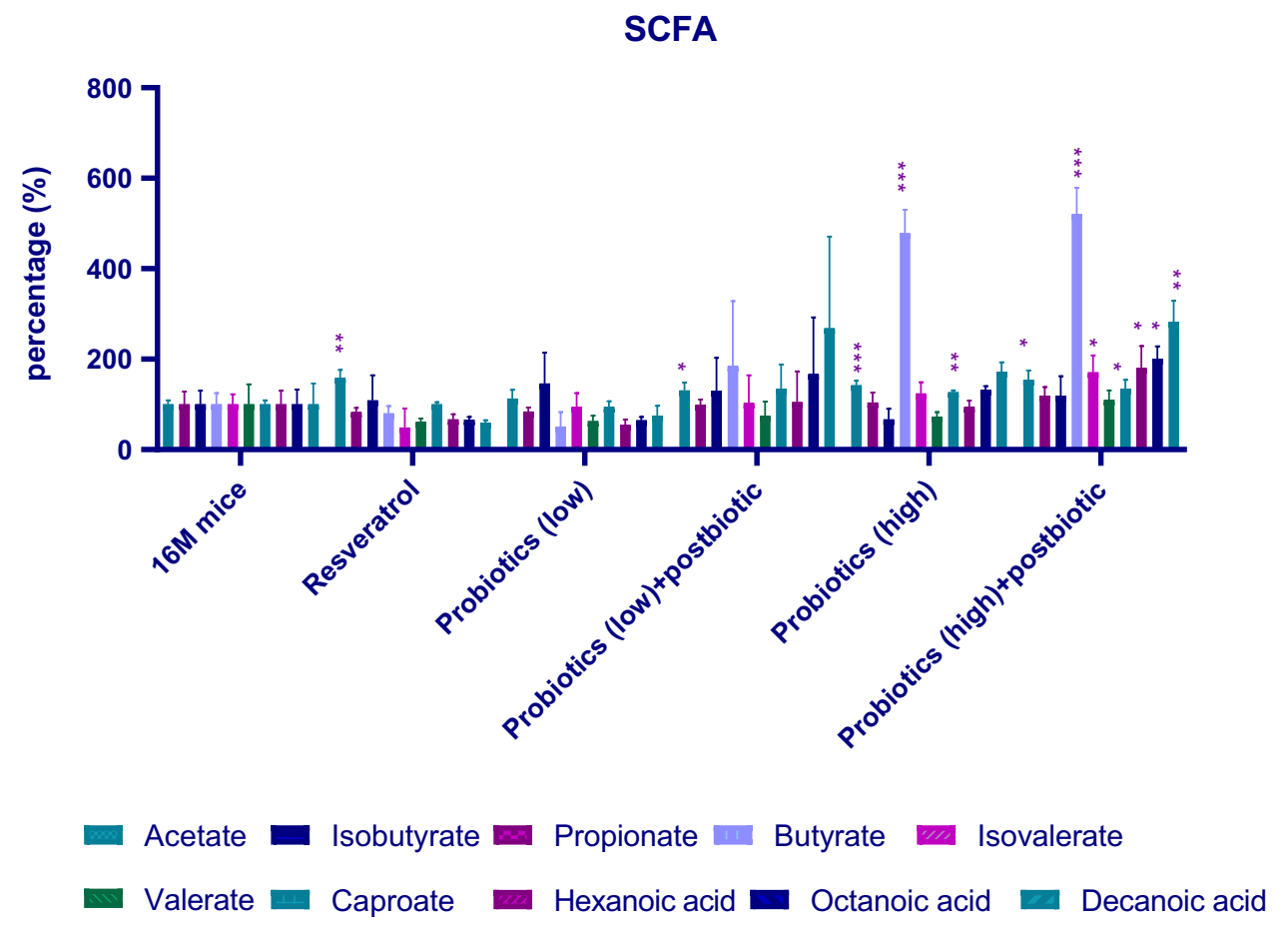

\section{Discussion}

In this study, we used a natural aging method to establish a 16-month-old murine model. Numerous studies have administrated D-galactose to establish an aging mice model, which successfully accelerated aging in 45 days [20-22].

Navarro et al. indicated that SOD levels and behavioral activity are significantly decreased in aging mice, whereas levels of the oxidative marker MDA are significantly increased in the mouse brain [23], which consistent with our natural aging model. Furthermore, levels of oxidative stress markers including MDA), protein carbonyl after protein oxidation, and mitochondrial $8 \mathrm{OHdG}$ decreased in mice receiving the probiotics (Fig. 2; Supplemental Figs, 2-5).

Mitochondria play an essential role in energy production through oxidative phosphorylation and intracellular homeostasis. Damage to or dysfunction of mitochondria can lead to aging, cardiovascular disease, neurodegenerative disorders, and cancer, among other outcomes [24]. Thus, the predominant forms of the free radical-induced oxidative marker mitochondrial DNA 8OHdG have been widely used as a biomarker of oxidative stress [25]. Our results indicate that mixed probiotic strains significantly reduced mitochondria $80 \mathrm{HdG}$ levels in the brain, liver, and kidney (Supplemental Figs, 2-5). However, there was no large difference between the number of probiotics in the low-dose group $\left(1.03 \times 10^{9} \mathrm{CFU} / \mathrm{kg}\right)$ and the high-dose group $\left(4.1 \times 10^{9} \mathrm{CFU} / \mathrm{kg}\right)$ in antioxidative activities. More probiotic dosages should be test in the antioxidative assays in the future.
Postbiotics are fermentation components generated by bioactive probiotic strains. They consist of various metabolites including microbial cell fractions, short-chain fatty acids, teichoic acid, extracellular polysaccharides, peptidoglycan-derived muropeptides, and functional proteins, and they reportedly benefit the regulation of anti-inflammatory and immune effects [26]. The synergetic effects of $L$. plantarum and $\beta$-glucans were reported to enhance digestive enzyme activity and intestinal morphology [27]. Our results also confirmed the synergetic effects of viable probiotic strains combined with their postbiotics in elevating antioxidative activities in the brain, liver, heart, and kidney of middle-aged mice (Fig. 2; Supplemental Figs. 2-5).

Gut microbiota have been recognized as playing a key role in aging and in antiaging interventions [28]. Evidence of the potential beneficial effects of dietary probiotics in older adults continues to accumulate [29]. In our study, compared with the young group, the proportions of the Bacteroidates phylum, Verrucomicrobia, and Actinobacteria were smaller in middle-aged mice, whereas the amount of Firmicutes was elevated (Fig. 3a) The genera of Lactobacillus, Bifidobacterium, and Akkermansia exhibited a similar tendency after treatment with probiotic formulas (Fig. 3b). The probiotic-fed groups had increased gut $B$. animalis, $B$. breve, $B$. bifidum, and $L$. plantarum, especially the highdose probiotics plus postbiotics group, which implies the fed probiotic strains successfully colonized the GI tract and enlarged the populations of the original species (Fig. 4a, b). Unexpectedly, the populations of some species not members of the fed probiotics also increased in the gut, including $L$. 
johnsonii, and Akkermansia muciniphila (Fig. 4a; Supplemental Fig. 6).

The abundance of $L$. johnsonii notably increased to $4.42 \%$ in the gut of mice receiving probiotic (high-dose) plus postbiotics (Fig. 4c). Several beneficial functions of $L$. johnsonii have been reported, including thickened mucous membranes in stomach ulcers [30], restored numbers of serum IgA, IgG, and CD8 + cells, increased splenocyte counts in aged mice with protein-energy malnutrition [31], decreased glucagon and glucose levels in diabetic rats [32], and prevention of memory dysfunction [33]. The proportion of gut Akkermansia was also significantly elevated, to $0.41 \%$, in mice receiving probiotics (high-dose) plus postbiotics, whereas in the 16-month-old mice it was only $0.04 \%$ (Fig. 4b). The colonization of Akkermansia in the gut was reported to relieve appendicitis-related inflammation and inflammatory bowel disease [34]. A recent clinical study discovered that Akkermansia were significantly increased among semisupercentenarians (age 105-109 years), which suggests that Akkermansia might play a part in building new gut homeostasis in extreme aging people [35].

A dysfunction in the gut-brain axis has been explained by a series of studies linked to neuropsychological, metabolic, and gastrointestinal disorders. Study findings reveal that perturbations of short-chain fatty acid and amino acid metabolism in serum and CSF are implicated in the onset of depression [36]. At the present study, the high-dose probiotics and high-dose probiotics plus postbiotics would significantly elevate serum butyrate levels (Fig. 5). Butyrate, a four-carbon short-chain fatty acid, is a crucial energy source for gut [37]. Studies had revealed multiple benefits of butyrate in human including enhancement of intestinal barrier function and mucosal immunity [38], elevating antiinflammation status [39], modulating oxidative stress in the colonic mucosa [40] and alleviation of depression-related symptoms [41]. Thus, the probiotic secreting SCFAs may be the key factors in antiaging effect.

In conclusion, the mixed probiotic formula of $B$. animalis subsp. infantis BLI-02, B. breve Bv889, B. bifidum VDD088VDD088, B. animalis subsp. lactis CP-9, and $L$. plantarum PL-02 successfully elevated antioxidative activity with positive modulation of beneficial intestinal microbiota and elevated serum SCFA in the middle-aged mice. However, the sample sizes of each experimental group should be enlarged to eight mice, and animal behavior testing should be performed in the future. Finally, a human study on antiaging function could be conducted using the mixed probiotic formula of this study [42].

Supplementary Information The online version contains supplementary material available at https://doi.org/10.1007/s00284-022-02783-y.
Acknowledgements Thanks to National Laboratory Animal Center (Taipei, Taiwan) for executing animal experiments. We also appreciated Wallace Academic Editing for editing this manuscript.

Author Contributions Conceptualization, HHH; validation, JHL and YWK; formal analysis, JHL; investigation, JHL, YWK, and RPFC; data curation, WYL; writing - original draft preparation, WYL; visualization, WYL, JHL, YWK, and HHH; Review and Editing, RPFC and WYL. All authors have read and agreed to the published version of the manuscript.

Funding This research did not receive any specific grant from funding agencies in the public, commercial, or not-for-profit sectors.

Data Availability Not applicable.

\section{Declarations}

Conflict of interest The authors declare no conflict of interest.

Ethical Approval Animal experiments and protocols were in compliance with the National Institute of Health's Guide for the Care and Use of Laboratory Animals. The protocols were approved (the Approval Number-IACUC no.NLAC(TN)-108-D-002) by animal ethics committee of National Laboratory Animal Center (Taipei, Taiwan).

Informed Consent Not applicable.

Open Access This article is licensed under a Creative Commons Attribution 4.0 International License, which permits use, sharing, adaptation, distribution and reproduction in any medium or format, as long as you give appropriate credit to the original author(s) and the source, provide a link to the Creative Commons licence, and indicate if changes were made. The images or other third party material in this article are included in the article's Creative Commons licence, unless indicated otherwise in a credit line to the material. If material is not included in the article's Creative Commons licence and your intended use is not permitted by statutory regulation or exceeds the permitted use, you will need to obtain permission directly from the copyright holder. To view a copy of this licence, visit http://creativecommons.org/licenses/by/4.0/.

\section{References}

1. López-Otín C, Blasco MA, Partridge L, Serrano M, Kroemer G (2013) The hallmarks of aging. Cell 153(6):1194-1217. https:// doi.org/10.1016/j.cell.2013.05.039

2. Blagosklonny MV (2008) Aging: ros or tor. Cell Cycle 7(21):3344-3354. https://doi.org/10.4161/cc.7.21.6965

3. Lin JK, Tsai SH (1999) Chemoprevention of cancer and cardiovascular disease by resveratrol. Life Sci 23(3):99-106. https://doi. org/10.1016/j.bbadis.2014.11.004

4. Fraga CG, Shigenaga MK, Park JW, Degan P, Ames BN (1990) Oxidative damage to DNA during aging: 8-hydroxy-2'-deoxyguanosine in rat organ DNA and urine. Proc Natl Acad Sci 87(12):4533-4537. https://doi.org/10.1073/pnas.87.12.4533

5. Thursby E, Juge N (2017) Introduction to the human gut microbiota. Biochem J 474(11):1823-1836. https://doi.org/10.1042/ BCJ20160510

6. Arboleya S, Watkins C, Stanton C, Ross RP (2016) Gut bifidobacteria populations in human health and aging. Front Microbiol 7:1204. https://doi.org/10.3389/fmicb.2016.01204 
7. Patel PJ, Singh SK, Panaich S, Cardozo L (2014) The aging gut and the role of prebiotics, probiotics, and synbiotics: a review. J Clin Gerontol Geriatr 5(1):3-6. https://doi.org/10.1016/j.jcgg. 2013.08.003

8. Kim H, Kim JS, Kim Y, Jeong Y, Kim JE, Paek NS, Kang CH (2020) Antioxidant and probiotic properties of lactobacilli and bifidobacteria of human origins. Biotechnol Bioprocess Eng 25(3):421-430. https://doi.org/10.1007/s12257-020-0147-x

9. Izuddin WI, Humam AM, Loh TC, Foo HL, Samsudin AA (2020) Dietary postbiotic Lactobacillus plantarum Improves serum and ruminal antioxidant activity and upregulates hepatic antioxidant enzymes and ruminal barrier function in post-weaning lambs. Antioxidants 9(3):250. https://doi.org/10.3390/antiox9030250

10. Akbari E, Asemi Z, Daneshvar Kakhaki R, Bahmani F, Kouchaki E, Tamtaji OR, Salami M (2016) Effect of probiotic supplementation on cognitive function and metabolic status in Alzheimer's disease: a randomized, double-blind and controlled trial. Front Aging Neurosci 8:256. https://doi.org/10.3389/fnagi.2016.00256

11. Saarela M, Mogensen G, Fonden R, Mättö J, Mattila-Sandholm T (2000) Probiotic bacteria: safety, functional and technological properties. J Biotechnol 84(3):197-215. https://doi.org/10.1016/ S0168-1656(00)00375-8

12. Ramírez-Garza SL, Laveriano-Santos EP, Marhuenda-Muñoz M, Storniolo CE, Tresserra-Rimbau A, Vallverdú-Queralt A, Lamuela-Raventós RM (2018) Health effects of resveratrol: results from human intervention trials. Nutrients 10(12):1892. https://doi.org/10.3390/nu10121892

13. Chiba T, Kimura Y, Suzuki S, Tatefuji T, Umegaki K (2016) Trans-resveratrol enhances the anticoagulant activity of warfarin in a mouse model. J Atheroscler Thromb. https://doi.org/10. 5551/jat.31765

14. Clarke G, Ting KN, Wiart C, Fry J (2013) High correlation of 2, 2-diphenyl-1-picrylhydrazyl (DPPH) radical scavenging, ferric reducing activity potential and total phenolics content indicates redundancy in use of all three assays to screen for antioxidant activity of extracts of plants from the Malaysian rainforest. Antioxidants 2(1):1-10. https://doi.org/10.3390/antiox2010001

15. Popović M, Kaurinović B, Trivić S, Mimica-Dukić N, Bursać M (2006) Effect of celery (Apium graveolens) extracts on some biochemical parameters of oxidative stress in mice treated with carbon tetrachloride. Phytother Res 20(7):531-537. https://doi. org/10.1002/ptr.1871

16. Gwak HJ, Rho M (2020) Data-driven modeling for species-level taxonomic assignment from 16S rRNA: application to human microbiomes. Front Microbiol 11:2866. https://doi.org/10.3389/ fmicb.2020.570825

17. Huang WC, Hsu YJ, Huang CC, Liu HC, Lee MC (2020) Exercise training combined with Bifidobacterium longum OLP-01 supplementation improves exercise physiological adaption and performance. Nutrients 12(4):1145. https://doi.org/10.3390/ nu 12041145

18. Shoji H, Takao K, Hattori S, Miyakawa T (2016) Age-related changes in behavior in C57BL/6J mice from young adulthood to middle age. Mol Brain 9(1):1-18. https://doi.org/10.1186/ s13041-016-0191-9

19. Flurkey K, Currer JM, Harrison DE (2007) Mouse models in aging research. The mouse in biomedical research. Academic Press, Cambridge, pp 637-672

20. Chen P, Chen F, Zhou BH (2019) Leonurine ameliorates $\mathrm{D}$-galactose-induced aging in mice through activation of the Nrf2 signalling pathway. Aging 11(18):7339. https://doi.org/ 10.18632/aging.101733

21. Ma K, Wu A, Yang T, Sheng D, Long C, Li L, Liu K (2014) Progressive impairment of motor skill learning in a D-galactoseinduced aging mouse model. Pak J Zool 46(1):215-221
22. Ho SC, Liu JH, Wu RY (2003) Establishment of the mimetic aging effect in mice caused by D-galactose. Biogerontology 4(1):15-18. https://doi.org/10.1023/a:1022417102206

23. Navarro A, Sánchez Del Pino MJ, Gómez C, Peralta JL, Boveris A (2002) Behavioral dysfunction, brain oxidative stress, and impaired mitochondrial electron transfer in aging mice. Am J Physiol-Regul Integr Comp Physiol 282(4):R985-R992. https:// doi.org/10.1152/ajpregu.00537.2001

24. Haas RH (2019) Mitochondrial dysfunction in aging and diseases of aging. Biology 8(2):48. https://doi.org/10.3390/biolo gy8020048

25. Valavanidis A, Vlachogianni T, Fiotakis C (2009) 8-hydroxy2'-deoxyguanosine (8-OHdG): a critical biomarker of oxidative stress and carcinogenesis. J Environ Sci Health C 27(2):120139. https://doi.org/10.1080/10590500902885684

26. Wegh CA, Geerlings SY, Knol J, Roeselers G, Belzer C (2019) Postbiotics and their potential applications in early life nutrition and beyond. Int J Mol Sci 20(19):4673. https://doi.org/10.3390/ ijms20194673

27. Dawood MA, Magouz FI, Salem MF, Elbialy ZI, Abdel-Daim HA (2019) Synergetic effects of Lactobacillus plantarum and $\beta$-glucan on digestive enzyme activity, intestinal morphology, growth, fatty acid, and glucose-related gene expression of genetically improved farmed tilapia. Probiotics Antimicrob Proteins. https://doi.org/10.1007/s12602-019-09552-7

28. Vaiserman AM, Koliada AK, Marotta F (2017) Gut microbiota: a player in aging and a target for anti-aging intervention. Ageing Res Rev 35:36-45. https://doi.org/10.1016/j.arr.2017.01.001

29. Landete JM, Gaya P, Rodríguez E, Langa S, Peirotén Á, Medina M, Arqués JL (2017) Probiotic bacteria for healthier aging: immunomodulation and metabolism of phytoestrogens. Biomed Res Int. https://doi.org/10.1155/2017/5939818

30. Pantoflickova D, Corthesy-Theulaz I, Dorta G, Stolte M, Isler P, Rochat F, Blum AL (2003) Favourable effect of regular intake of fermented milk containing Lactobacillus johnsonii on Helicobacter pylori associated gastritis. Aliment Pharmacol Ther 18(8):805-813. https://doi.org/10.1046/j.1365-2036.2003.01675.x

31. Kaburagi T, Yamano T, Fukushima Y, Yoshino H, Mito N, Sato K (2007) Effect of Lactobacillus johnsonii La1 on immune function and serum albumin in aged and malnourished aged mice. Nutrition 23(4):342-350. https://doi.org/10.1016/j.nut.2007.02.001

32. Yamano T, Tanida M, Niijima A, Maeda K, Okumura N, Fukushima Y, Nagai K (2006) Effects of the probiotic strain Lactobacillus johnsonii strain La1 on autonomic nerves and blood glucose in rats. Life Sci 79(20):1963-1967. https://doi.org/10.1016/j.lfs. 2006.06.038

33. Wang H, Sun Y, Xin J, Zhang T, Sun N, Ni X, Bai Y (2020) Lactobacillus johnsonii BS15 prevents psychological stress-induced memory dysfunction in mice by modulating the gut-brain axis. Front Microbiol 11:1941. https://doi.org/10.3389/fmicb.2020. 01941

34. Van Passel MW, Kant R, Zoetendal EG, Plugge CM, Derrien M, Malfatti SA, Smidt H (2011) The genome of Akkermansia muciniphila, a dedicated intestinal mucin degrader, and its use in exploring intestinal metagenomes. PLoS ONE 6(3):e16876. https://doi. org/10.1371/journal.pone.0016876

35. Biagi E, Franceschi C, Rampelli S, Severgnini M, Ostan R, Turroni S, Capri M (2016) Gut microbiota and extreme longevity. Curr Biol 26(11):1480-1485. https://doi.org/10.1016/j.cub.2016. 04.016

36. Deng FL, Pan JX, Zheng P, Xia JJ, Yin BM, Liang WW, Xie P (2019) Metabonomics reveals peripheral and central short-chain fatty acid and amino acid dysfunction in a naturally occurring depressive model of macaques. Neuropsychiatr Dis Treat 15:1077. https://doi.org/10.2147/NDT.S186071 
37. Hamer HM, Jonkers DMAE, Venema K, Vanhoutvin SALW, Troost FJ, Brummer RJ (2008) The role of butyrate on colonic function. Aliment Pharmacol Ther 27(2):104-119. https://doi.org/ 10.1111/j.1365-2036.2007.03562.x

38. Bach Knudsen KE, Lærke HN, Hedemann MS, Nielsen TS, Ingerslev AK, Gundelund Nielsen DS, Hermansen K (2018) Impact of diet-modulated butyrate production on intestinal barrier function and inflammation. Nutrients 10(10):1499. https://doi.org/10.3390/ nu10101499

39. Hamer HM, Jonkers DM, Vanhoutvin SA, Troost FJ, Rijkers G, de Bruïne A, Brummer RJM (2010) Effect of butyrate enemas on inflammation and antioxidant status in the colonic mucosa of patients with ulcerative colitis in remission. Clin Nutr 29(6):738744. https://doi.org/10.1016/j.clnu.2010.04.002

40. Hamer HM, Jonkers DM, Bast A, Vanhoutvin SA, Fischer MA, Kodde A, Brummer RJM (2009) Butyrate modulates oxidative stress in the colonic mucosa of healthy humans. Clin Nutr 28(1):88-93. https://doi.org/10.1016/j.clnu.2008.11.002
41. Tian P, Wang G, Zhao J, Zhang H, Chen W (2019) Bifidobacterium with the role of 5-hydroxytryptophan synthesis regulation alleviates the symptom of depression and related microbiota dysbiosis. J Nutr Biochem 66:43-51. https://doi.org/10.1016/j.jnutb io.2019.01.007

42. Lee DE, Huh CS, Ra J, Choi ID, Jeong JW, Kim SH, Sim JH (2015) Clinical evidence of effects of Lactobacillus plantarum HY7714 on skin aging: a randomized, double blind, placebo-controlled study. J Microbiol Biotechnol 25(12):2160-2168. https:// doi.org/10.4014/jmb.1509.09021

Publisher's Note Springer Nature remains neutral with regard to jurisdictional claims in published maps and institutional affiliations. 